\title{
Aspect-ratio-dependent charging in high-density plasmas
}

\author{
Gyeong S. Hwang and Konstantinos P. Giapis ${ }^{\text {a) }}$ \\ Division of Chemistry and Chemical Engineering, California Institute of Technology, Pasadena, \\ California 91125
}

(Received 18 December 1996; accepted 18 April 1997)

\begin{abstract}
The effect of aspect ratio (depth/width) on charge buildup in trenches during plasma etching of polysilicon-on-insulator structures is studied by Monte Carlo simulations. Increased electron shadowing at larger aspect ratios reduces the electron current to the trench bottom. To reach a new charging steady state, the bottom potential must increase, significantly perturbing the local ion dynamics in the trench: the deflected ions bombard the sidewall with larger energies resulting in severe notching. The results capture reported experimental trends and reveal why the increase in aspect ratio that follows the reduction in critical device dimensions will cause more problems unless the geometry is scaled to maintain a constant aspect ratio. (C) 1997 American Institute of Physics. [S0021-8979(97)06414-1]
\end{abstract}

\section{INTRODUCTION}

The incessant reduction in the lateral dimensions of integrated circuits has significantly increased the aspect ratio (AR) of device features which, in turn, has created serious new problems for semiconductor processing technology. In reactive ion etching of gate electrodes and contact windows, for example, it was soon discovered that the etch rate decreases with the AR. ${ }^{1}$ High AR trenches did not clear as fast as more open areas, thus requiring an overetch step that exposed the cleared layers longer to a potentially damaging plasma. Moreover, linewidth loss in the smaller devices became intolerable, requiring operation at lower pressures to improve anisotropy. Since conventional rf plasmas did not operate efficiently at low pressures, new high-density plasma sources were introduced, ${ }^{2}$ enabling the etching of high AR features without compromising anisotropy or etch rate. High throughput was achieved by the ability of high-density plasmas to deliver large ion fluxes to the wafer. However, the advantageous larger ion and electron current densities to the wafer, when combined with high AR structures, brought about the possibility of significant microstructure charging and a new form of plasma process-induced damage. ${ }^{3-5}$

When bombarded by unequal fluxes of ions and electrons, insulating surfaces will charge up. Such surfaces are ubiquitous on wafers during plasma etching. In gate electrode definition, for example, gate oxide, polycrystalline silicon (poly-Si gate), and photoresist may all be exposed to the plasma simultaneously. The directionality difference between ions (anisotropic) and electrons (isotropic) at the wafer surface, created as they cross the sheath, will then cause enhanced electron accumulation at the upper photoresist sidewalls confining a narrow trench, while ions bombard the exposed $\mathrm{SiO}_{2}$ surface at the trench bottom. The repulsive entrance potential reduces the flux of electrons to the trench bottom, thus forcing the $\mathrm{SiO}_{2}$ surface potential to increase, until enough ions are deflected so that the ion and electron fluxes are balanced. ${ }^{6,7}$ More deflected ions bombard the inner

\footnotetext{
a) To whom correspondence should be addressed. Electronic mail: giapis@cheme.caltech.edu
}

side of the edge gate of a dense line-and-space pattern because its potential can be maintained low by electrons arriving at its outer sidewall from the open area. Geometric shadowing prohibits the latter from occurring at intermediate lines. Enhanced bombardment of the poly-Si sidewall foot leads to the so-called "notching" effect, ${ }^{3,4}$ describing a wedgelike profile irregularity, whose location and shape are depicted in Fig. 1. Since the poly-Si gate is isolated, the ion accumulation increases its potential. If the potential difference between gate electrode and substrate becomes excessive, large electric fields in the gate oxide may induce Fowler-Nordheim $(\mathrm{F}-\mathrm{N})$ tunneling currents, which can cause oxide degradation or even breakdown. ${ }^{5}$ Both, F-N tunneling currents and notching constitute charging damage, a problem that has puzzled the etching community for many years and is feared to become a show stopper at critical dimensions of $0.18 \mu \mathrm{m}$ and beyond.

Understanding charging damage requires comprehensive modeling and simulation of ion and electron dynamics in plasmas, through sheaths, and as they impinge at various microstructure surfaces where they cause charging, physical sputtering, and chemical reactions, or undergo inelastic scattering. A predictive feature-scale simulation that discusses these phenomena and simplified models to describe them have been presented elsewhere. ${ }^{7}$ In this article, we expand our simulation to reveal the influence of the aspect ratio on pattern-dependent charging and related profile distortion effects. In previous experimental work, Hashimoto ${ }^{5}$ has shown that the catastrophic breakdown of metal-oxidesemiconductor (MOS) capacitors (antenna structures) increases with the photoresist AR. Kamata and Arimoto ${ }^{8}$ measured a decrease in electron current to the substrate following an increase in the AR of a $\mathrm{SiO}_{2}$ mask. Fujiwara et al. ${ }^{4}$ have also shown a monotonic but complex increase in notch depth (a signature of charging damage) with an increase of the AR in the regime between 0.7:1 and 2.8:1. While it is anticipated that notching will further worsen at higher aspect ratios, no modeling study has appeared to provide insight into why and how charging in the trench is affected by the aspect ratio. 


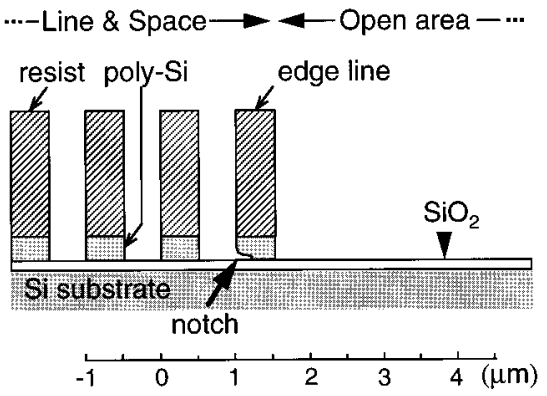

FIG. 1. Schematic depiction of the line-and-space structure considered in the simulation. The location of the notch is also shown. The photoresist thickness is varied from 0.2 to $1.7 \mu \mathrm{m}$ to change the aspect ratio. The case depicted corresponds to an aspect ratio of $4: 1$.

\section{PLASMA CONDITIONS AND MICROSTRUCTURE DESCRIPTION}

The plasma properties influence microstructure charging profoundly. Here, we employ a typical chlorine plasma with a density of $1 \times 10^{12} \mathrm{~cm}^{-3}$. The wafer electrode is biased and the sheath voltage, $V_{s}$, is determined from $V_{s}=37+30 \sin \omega t \mathrm{~V}$, where $\omega=400 \mathrm{kHz}$ is the rf bias frequency. The ion and electron temperatures are assumed to be 0.5 and $4 \mathrm{eV}$, respectively. The pressure is taken to be low enough ( $<10$ mTorr) to allow for ballistic transport through the sheath and in the microstructure spaces. The chlorine is assumed to be $100 \%$ dissociated at the relatively high plasma density considered here.

The dense structure of Fig. 1 borders a widely open area and consists of gratings with $0.5 \mu \mathrm{m}$ lines separated by 0.5 $\mu \mathrm{m}$ spaces (trenches); the lines are not electrically connected. The simulation starts with a perfectly etched pattern of lines, each consisting of insulating photoresist onto 0.3 $\mu \mathrm{m}$ poly-Si; the features are formed on top of a uniform layer of $\mathrm{SiO}_{2}$, thick enough $(>100 \mathrm{~nm})$ to prevent tunneling currents to the Si substrate. The height of the photoresist will be varied so that the AR spans the regime from 1:1 to 4:1. The height of the poly-Si remains constant. When the $\mathrm{AR}=4$, the features consist of a $1.7 \mu \mathrm{m}$ photoresist onto 0.3 $\mu \mathrm{m}$ poly-Si, clearly not representative of current device manufacturing technology. However, in addition to being instructive, consideration of such a case may not be as extreme at smaller critical dimensions; for the $0.18 \mu \mathrm{m}$ device rule, for example, a very reasonable photoresist thickness of 0.54 $\mu \mathrm{m}$ on top of a $0.18 \mu \mathrm{m}$ poly-Si would produce an $\mathrm{AR}=4$. The poly-Si is assumed to be heavily doped (electrically conductive). The profile evolution of the inner sidewall of the edge line during overetching will be simulated.

\section{SIMULATION OF MICROSTRUCTURE CHARGING}

Microstructure charging is performed by a Monte Carlo simulation of ion and electron transport first through a collisionless sheath and then through the microstructure spaces, as described elsewhere. ${ }^{7}$ Briefly, realistic ion and electron energy and angular distributions at the wafer are calculated from sheath theory. ${ }^{9}$ Then, charged particles are followed as they impinge on various surfaces, where they transfer their

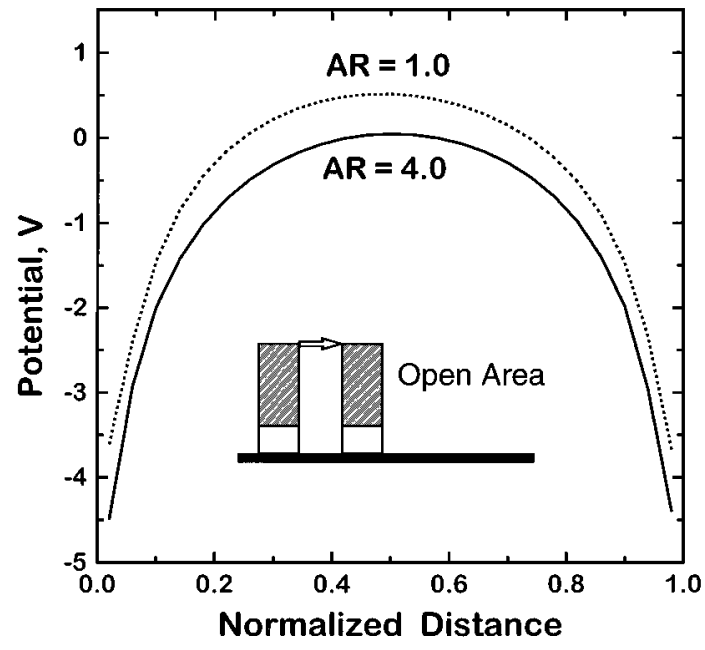

FIG. 2. The potential distribution along the line connecting the top corners of the photoresist sidewalls that border the trench by the edge line, as indicated at the inset (arrow). The distance has been normalized by the trench width $(0.5 \mu \mathrm{m})$.

charge. Charge deposition creates local electric fields which, in turn, alter ion trajectories. The Laplace equation is solved iteratively in the microstructure spaces to account for the evolution of the electric fields as more charge accumulates, until steady state is reached. Reactions at the sidewalls during the charging process are neglected, which is a reasonable assumption in view of the rapid charge buildup. The mobility of charges in conductive materials (doped poly-Si) influences the charging process critically. All surfaces of such materials must reach equipotential; in the presence of strong local electric fields, this condition requires redistribution of charge from the points of accumulation to all surfaces. Steady state is established when the potential distribution along the bottom $\mathrm{SiO}_{2}$ surface no longer changes. The potential steady state requires a significantly longer charging time than that needed to balance the electron and ion currents at the bottom $\mathrm{SiO}_{2}$ surface. ${ }^{7}$ For the present calculation, surface currents and secondary electron emission are both neglected. As discussed earlier, tunneling currents through the thick gate oxide are also disregarded; thus, the present study focuses on a worst-case scenario, aiming to reveal where breakdown is most likely to occur from excessive charging.

For a given design rule, a larger aspect ratio implies that the negatively charged upper photoresist sidewalls are further removed from the positively charged bottom $\mathrm{SiO}_{2}$ surface. Increasing the distance between the two charged surface segments weakens the dipole field in the trench. The entrance potential distribution becomes more negative-by about half a volt when increasing the AR from 1 to 4 -thus increasing the number of low energy electrons that are repelled back into the plasma. This is demonstrated by plotting the potential between the photoresist sidewalls along the line that connects the top corners, as shown in Fig. 2. Thus, the electron current into the trench is reduced. The longer sidewalls at the larger AR also attract electrons, further depleting the electron flux to the trench bottom. This result is illustrated in Fig. 3, where the normalized electron fluxes to vari- 


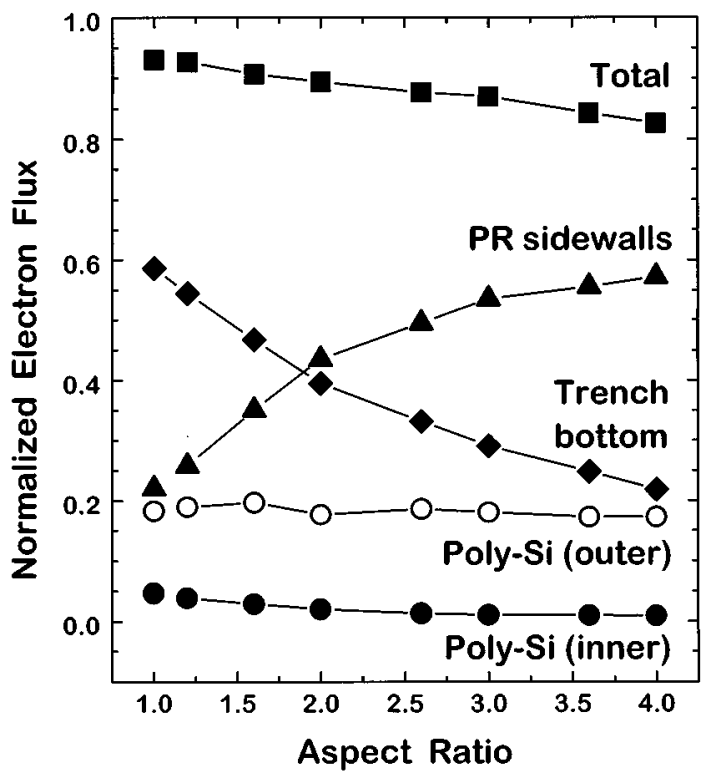

FIG. 3. The normalized electron flux into the trench by the edge line (total), to both photoresist (PR) sidewalls that border the trench, to the bottom $\mathrm{SiO}_{2}$ surface, and to the inner and outer poly-Si sidewalls of the edge line, after steady-state charging has been reached, as a function of the aspect ratio.

ous surfaces in the trench are plotted as a function of the AR. Clearly, the electron flux to the bottom $\mathrm{SiO}_{2}$ surface decreases much more than the flux of electrons entering the trench, as a result of the presence of the longer insulating sidewalls. This form of shadowing is not strictly geometric, since the reduction in electron current to the trench bottom is calculated to be significantly smaller than that predicted by the decrease in the solid angle that "views" the plasma from the trench bottom. Electrostatics plays a vital role in decreasing the geometric shadowing, as explained in the following paragraph. Remarkably, both the electron flux to the inner and outer poly-Si sidewall of the edge line change only slightly with the AR, an observation with important implications for inner sidewall distortion (notching).

The reduction in electron current to the trench bottom at a larger AR leads to a larger surface potential on that surface, which causes more ions to be deflected towards the sidewalls. These results are quantified in Fig. 4, where the normalized ion flux to both the trench bottom and the inner poly-Si sidewall of the edge line are plotted as a function of the AR. Indeed, the ion current to the trench bottom decreases by about $60 \%$, when the AR is increased from 1 to 4 . As expected, this curve is identical to the one of Fig. 3 that describes the electron current to the trench bottom. Surprisingly, the flux of deflected ions that impinge on the inner poly-Si sidewall of the edge line does not change significantly-in fact, it decreases slightly! This result is consistent with the calculated electron fluxes to the inner and outer poly-Si sidewalls of the edge line, which were also not significantly affected by the increase in the aspect ratio. So, where do the deflected ions go and why does notching become more severe for larger aspect ratios?

It was found earlier that the flux of electrons to the photoresist sidewalls increases substantially with the AR. To

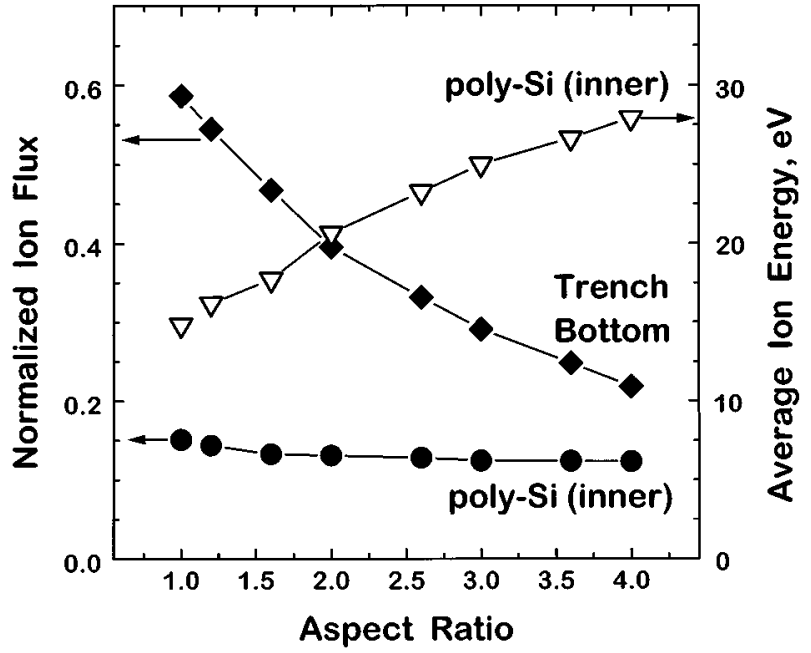

FIG. 4. The normalized flux of ions arriving at the trench bottom and the inner poly-Si sidewall of the edge line after steady-state charging has been reached, as a function of the aspect ratio. The average incident energy of the ions impinging at the inner poly-Si sidewall of the edge line is also shown.

balance this current at steady state, a significant ion flux is required, which is supplied by the deflected ions from the trench bottom. The equipotential of the neighboring poly-Si line must increase simultaneously with the bottom potential in order to assist in the process of ion deflection towards the photoresist sidewalls above it. Note that some ions must even be deflected out of the trench to balance the reduced influx of electrons (vide supra).

In spite of the relatively constant ion flux to the inner poly-Si sidewall of the edge line, notching will worsen with increasing $\mathrm{AR}$, provided that the translational energy of the deflected ions becomes larger. Indeed, our calculations confirm the latter, as shown in Fig. 4. Even for an $A R=1$, the average energy is larger than the threshold for a reaction with a chlorinated poly-Si surface $(10 \mathrm{eV}) .{ }^{10}$ The average energy clearly increases with the AR, thus increasing the etch yield. Note that the rate of increase does not appear to saturate even for an $\mathrm{AR}=4$. However, sidewall distortion should not worsen indefinitely as the AR increases; electron shadowing stops increasing beyond a certain value of the AR, as new mechanisms for ion and electron transport through long holes with insulating walls take over. ${ }^{11}$ To understand why the average energy of the deflected ions increases, we must first elucidate how the ion dynamics in the trench are modified when the AR increases.

The steady-state charging potential distribution in the "edge" trench reveals the perturbation in the local ion dynamics occurring as a result of surface charging (Fig. 5). Gradients on this potential surface are a measure of the electric field that influences ion motion. The asymmetric potential distribution at the trench bottom, with the pronounced peak near the inner sidewall foot of the outermost line, is a sine qua non for the formation of the notch. ${ }^{12}$ Notice the appearance of a second smaller peak in the potential distribution near the sidewall foot of the neighboring line, most obvious for an $\mathrm{AR}=1$ [Fig. 5(a)]. This peak appears because of the low equipotential of the corresponding poly-Si line for 

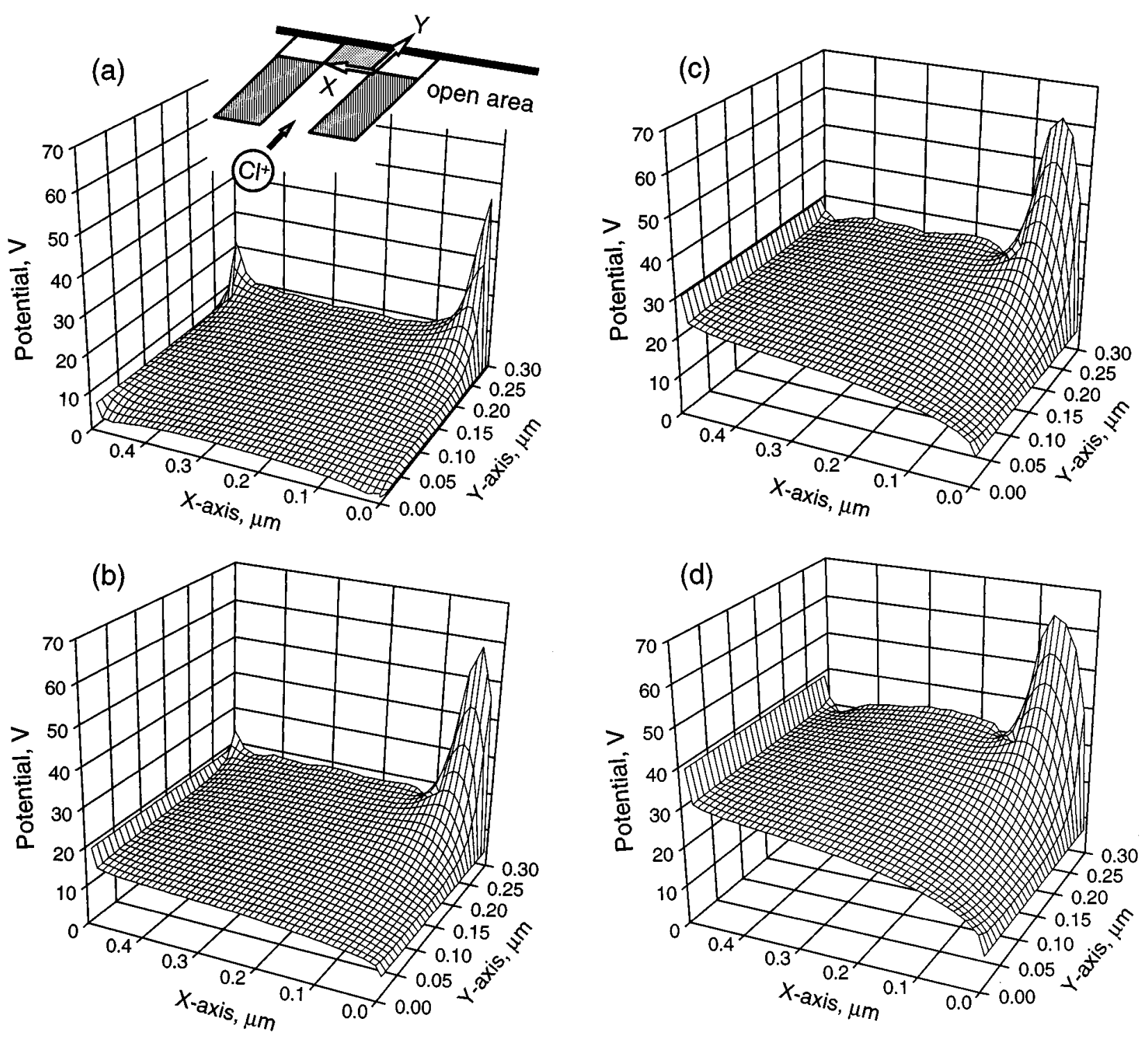

FIG. 5. Three-dimensional charging potential distributions in the trench area bordered by the poly-Si sidewalls, for structures with an aspect ratio of (a) 1:1, (b) 2:1, (c) 3:1, and (d) 4:1, after steady-state charging has been reached. The inset in (a) illustrates the area of interest and defines the origin for the potential surface (aspect ratio not to scale). The microstructure has been rotated to allow for a more convenient description of ion motion in the trench. The arrow shows the direction of ions as they approach the potential surface.

an $\mathrm{AR}=1$; however, it is not expected to cause a significant profile distortion in view of the weak local electric field it creates. The magnitude of the maximum in the potential by the edge line depends weakly on the AR and only for an $\mathrm{AR} \leqslant 2$; it is affected mainly by the rf bias and frequency. However, the charging potential distribution in the trench space away from the peak value increases significantly with increasing AR. For example, the potential at the center of the bottom surface increases from approximately 8 to $33 \mathrm{~V}$, when the AR is increased from 1 to 4 , respectively [Fig. 5(d)]. As discussed earlier, this behavior is necessary to balance the ion and electron currents to the bottom surface. The sidewall potentials also increase, in such a way that the tilt of the potential surface in the trench space towards the outermost line actually increases. Thus, the ions that are deflected towards the inner side of the outermost poly-Si line will acquire a larger translational energy as they fall down a steeper potential gradient. It becomes obvious now why profile distortion and notching will worsen with an increasing aspect ratio. ${ }^{4}$

The perturbation in the ion dynamics in the trench culminates into an increase in the poly-Si equipotential of both the edge line and the neighboring line, following an increase in the AR. The outer sidewall of the edge line is supplied by electrons from the open space, which help prevent a significant increase in the corresponding potential. The same is not possible for the poly-Si sidewalls of the neighboring line, whose potential should increase much more than that of the edge line. Figure 6 illustrates the dependence of the equipotentials of both lines as a function of the AR. Indeed, the poly-Si equipotential of the neighboring line exhibits a steeper and much more significant rise with the AR than that of the edge line. The difference between the two equipotentials is a measure of the electric field that accelerates ions 


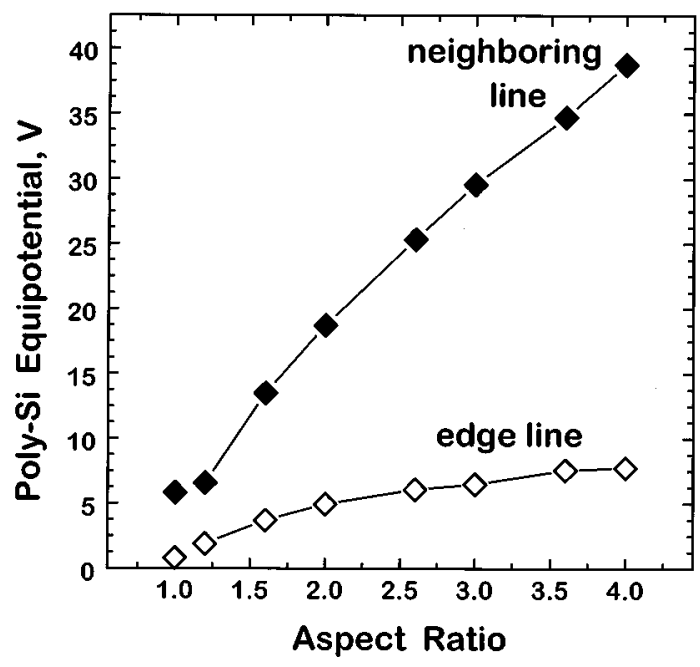

FIG. 6. The steady-state equipotentials of the poly-Si edge line and its neighboring line, as a function of the aspect ratio. For definitions, see Fig. 1.

towards the inner sidewall of the edge line; this difference increases with the AR, an observation consistent with the increase in the average energy of ions impinging onto the same sidewall, shown in Fig. 4. Note that Fig. 6 also suggests that catastrophic $\mathrm{F}-\mathrm{N}$ tunneling currents are more likely to occur under the gate electrode of the neighboring line, should thinner oxides be used. When the $\mathrm{AR}=4$, for example, a 5-nm-thick oxide layer will withstand the $5 \mathrm{~V}$ potential difference between the poly-Si edge line and the substrate, ${ }^{13}$ since the magnitude of the electric field in the oxide is smaller than the breakdown threshold $(1-1.2 \mathrm{~V} / \mathrm{nm})$. However, the potential difference across the gate oxide of the neighboring line is more than sixfold the threshold value, which implies that catastrophic failure of the oxide would have occurred long before this potential difference could be established.

\section{SIMULATION OF SIDEWALL PROFILE EVOLUTION}

The steady-state charging potentials determine the ion dynamics in the microstructure. Reactions at the poly-Si sidewalls and scattering at the $\mathrm{SiO}_{2}$ surface must be considered for the profile evolution simulation, which is based on a cell-removal algorithm and is performed as described elsewhere. ${ }^{7}$ As etching of the sidewall proceeds, charging of the exposed $\mathrm{SiO}_{2}$ leads to significant forward deflection of energetic ions that can contribute to etching. Thus, the etching simulation becomes coupled to transient charging in the etched area, further complicating the calculation. The problem becomes tractable by solving the Laplace equation each time the notch apex is advanced to the next cell layer and then using the calculated electrostatic force field to determine the ion trajectories for etching of the sidewall and charging of the bottom $\mathrm{SiO}_{2}$ surface. Etching of the poly-Si is assumed to be ion-limited while spontaneous etching by $\mathrm{Cl}$ atoms is neglected. Sputtering and chemical etching of $\mathrm{SiO}_{2}$ are presumed to be insignificant at the ion energies considered. A simple (square root) ion energy dependence of the poly-Si etch yield is assumed, which implies that a physi-

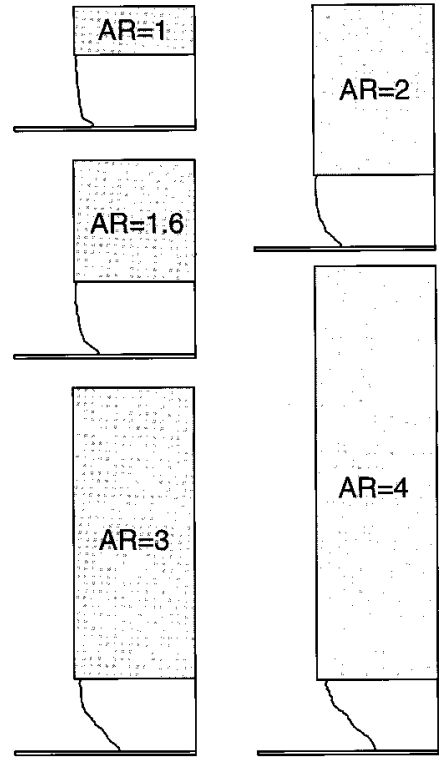

FIG. 7. Notch profiles of the inner sidewall of the edge line, predicted for $100 \%$ overetching of structures with increasing aspect ratio, as indicated. The shaded rectangles are the photoresist, the profiled area underneath is the poly-Si, and the thin double line represents the $\mathrm{SiO}_{2}$. The aspect ratio has been preserved to give a perspective of the structure. The evolution of the outer sidewall is not simulated.

cal sputtering mechanism is the prevalent mode by which material is removed from the surface. ${ }^{14}$ There is an energy threshold of $10 \mathrm{eV}$ for the reaction of $\mathrm{Cl}^{+}$with poly-Si, ${ }^{10}$ which influences sidewall evolution profoundly as it prevents a large number of low energy ions from contributing to the etching. The etch yield does not depend on the angle of incidence $\left(\theta_{i}\right)$ up to $45^{\circ}$; for larger $\theta_{i}$, it decreases as $\cos \theta_{i}{ }^{7}$ Inelastic scattering on poly-Si is neglected as most of the scattered atoms impinge subsequently on $\mathrm{SiO}_{2}$. However, direct inelastic scattering on the Cl-covered $\mathrm{SiO}_{2}$ surface must be included as all of the scattered atoms impinge subsequently on poly-Si. Only specular reflection is considered and the energy transfer is presumed to follow hardsphere collision kinematics ${ }^{14}$ with a gas atom to surface mass ratio of 1.0 .

Profile evolution simulations for line-and-space structures next to an open area have been performed, and the profiles obtained for $100 \%$ overetching are shown in Fig. 7 for various aspect ratios. As expected, increasing the AR leads to significantly deeper and wider notches. The profile for an $\mathrm{AR}=4$ exhibits the broadest and deepest notch, while it simultaneously shows substantial undercutting under the mask, a consequence of the increased energy of ions deflected towards the upper poly-Si sidewall. The undercutting is considerably reduced for an $\mathrm{AR} \leqslant 2$; for even smaller $\mathrm{AR}$, the notch gets narrower and more typical of the plasma conditions employed. ${ }^{3}$ Remarkably, a minuscule notch still exists for an $\mathrm{AR}=1$. Since $100 \%$ overetching is rather excessive at such low AR values, notching is not expected to be a serious problem in this case. Thus, decreasing the aspect ratio should help minimize plasma-induced charging problems. The current state-of-the-art fabrication technology, however, requires etching of larger AR features as devices get smaller; 


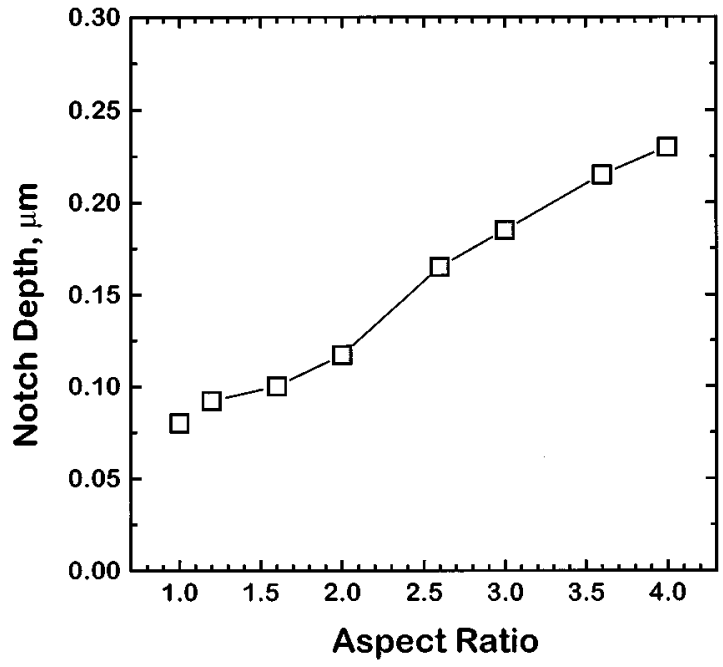

FIG. 8. Predicted notch depth dependence on aspect ratio. Some of the data were obtained from the profiles of Fig. 7.

therefore, solutions to the notching effect are needed, capable of alleviating the problem even for larger AR values.

The results of Fig. 7, together with calculations for additional structures, are summarized in Fig. 8, where the notch depth is plotted as a function of the AR. Whereas initially the dependence on the AR is weak, since electron shadowing is relatively unimportant, the rate of notch deepening increases significantly for an $\mathrm{AR}>2$. The notch depth is expected to approach a constant value for some $A R>4$ as electron and ion transport through the trench become independent of aspect ratio. ${ }^{11}$ These results are in good agreement with experimental trends observed in the work of Fujiwara et al. ${ }^{4}$

Finally, we wish to point out an important difference between the influence of aspect ratio versus electron temperature on notching. When both of these parameters are increased independently, the trench entrance potential gets more negative causing the bottom potential to increase. ${ }^{15}$ Notching worsens in both cases. ${ }^{4}$ When the aspect ratio increases, the average energy of the deflected ions that bombard the poly-Si sidewall of the edge line increases while the ion flux remains approximately constant. When the electron temperature increases-for constant, but high aspect ratio features, the average ion energy remains approximately constant while the deflected ion flux arriving at the sidewall increases. ${ }^{15}$

\section{SUMMARY AND CONCLUSIONS}

Detailed results from the Monte Carlo simulations of pattern-dependent charging and etching show that the aspect ratio critically influences charging damage during overetching of polysilicon-on-insulator structures in uniform highdensity plasmas. Increasing the aspect ratio worsens electron shadowing, which leads to a reduction in the electron current density to the trench bottom. To balance this current, the charging potential at the bottom $\mathrm{SiO}_{2}$ surface must increase so that the ion current density is reduced by the same amount. A larger repulsive potential at the trench bottom causes more low energy ions to be deflected; these ions are attracted by the upper photoresist sidewalls, where they balance the increased electron flux. Remarkably, the deflected ion flux towards the inner sidewall of the edge line in a line-and-space pattern remains approximately constant as the aspect ratio increases. However, the electric field towards the inner sidewall increases significantly, accelerating the deflected ions to larger translational energies which, in turn, cause the formation of a wider and deeper notch at that location. Although the results presented here were based on 0.5 $\mu \mathrm{m}$ design rule, they are also applicable to smaller design rules, as charging effects scale with aspect ratio. Thus, low aspect ratio structures are preferable from the standpoint of plasma-induced charging damage, albeit not practical at very small critical dimensions $(\leqslant 0.25 \mu \mathrm{m})$, where gate and photoresist thickness limitations exist. For example, at a 0.18 $\mu \mathrm{m}$ device rule, aspect ratios smaller than two seem unrealistic, even with a hard mask. Charging damage will persist in the next generations of devices, unless a way is found to reduce the charging potential at the trench bottom.

This work was partially supported by an NSF Career Award to K.P.G. (CTS-9623450).

${ }^{1}$ R. A. Gottscho, C. W. Jurgensen, and D. J. Vitkavage, J. Vac. Sci. Technol. B 10, 2133 (1992), and references therein.

${ }^{2}$ M. A. Lieberman and R. A. Gottscho, Design of High-Density Plasma Sources for Materials Processing, Physics of Thin Films Vol. 18, edited by M. H. Francombe and J. L. Vossen (Academic, New York, 1994).

${ }^{3}$ T. Nozawa, T. Kinoshita, T. Nishizuka, A. Narai, T. Inoue, and A. Nakaue, Jpn. J. Appl. Phys. 34, 2107 (1995).

${ }^{4}$ N. Fujiwara, T. Maruyama, and M. Yoneda, Jpn. J. Appl. Phys. 34, 2095 (1995); 35, 2450 (1996).

${ }^{5}$ K. Hashimoto, Jpn. J. Appl. Phys. 32, 6109 (1993); 33, 6013 (1994).

${ }^{6}$ T. Kinoshita, M. Hane, and J. P. McVittie, J. Vac. Sci. Technol. B 14, 560 (1996)

${ }^{7}$ G. S. Hwang and K. P. Giapis, J. Vac. Sci. Technol. B 15, 70 (1997).

${ }^{8}$ T. Kamata and H. Arimoto, J. Appl. Phys. 80, 2637 (1996).

${ }^{9}$ M. A. Lieberman and A. J. Lichtenberg, Principles of Plasma Discharges and Materials Processing (Wiley Interscience, New York, 1994).

${ }^{10}$ J. P. Chang and H. H. Sawin, J. Vac. Sci. Technol. A (to be published).

${ }^{11}$ G. S. Hwang and K. P. Giapis (unpublished).

${ }^{12}$ The difference between the potentials of the edge line (low) and the neighboring line (high) steers the ions approaching the trench bottom towards the low equipotential. Since more ions impinge on the bottom surface near the sidewall foot of the edge line, a larger positive potential builds up at that location, resulting in an asymmetric potential distribution at the trench bottom. The symmetric potential distribution calculated by Kinoshita et al. (Ref. 6) is not physical and suggests that notching should occur at both sidewalls confining the edge trench, contrary to experimental evidence (Refs. 3 and 4).

${ }^{13}$ This conjecture is based on maintaining the substrate potential close to $0 \mathrm{~V}$.

${ }^{14}$ G. S. Hwang, C. M. Anderson, M. J. Gordon, T. A. Moore, T. K. Minton, and K. P. Giapis, Phys. Rev. Lett. 77, 3049 (1996).

${ }^{15}$ G. S. Hwang and K. P. Giapis, J. Appl. Phys. 81, 3433 (1997). 